\title{
A NOTE ON POINTWISE CONVERGENCE
}

\author{
M. K. FORT, JR.
}

Let $C$ be the set of all continuous real-valued functions on the closed interval $[0,1]$. The question arises as to whether or not a metric $d$ for $C$ exists with the property that $\lim _{n \rightarrow \infty} d\left(f_{n}, f_{0}\right)=0$ if and only if the sequence $\left\{f_{n}\right\}$ converges pointwise to $f_{0}$. It is well known that such a metric $d$ does not exist, but a correct proof of the nonexistence of such a $d$ does not seem to be so well known.

The following false "proof" of the nonexistence of $d$ seems to be prevalent: "The weak topology for $C$ defines a notion of convergence which is pointwise convergence. The weak topology for $C$ does not satisfy the first countability axiom and consequently is not metrizable. Hence no metric for $C$ exists which defines convergence to be pointwise convergence." The fallacy in this line of reasoning lies in the fact that two topologies, one metrizable and the other nonmetrizable, can induce the same notion of convergence for sequences. ${ }^{1}$

We now construct a double sequence $\left\{f_{n, m}\right\}$ of elements of $C$. This double sequence is used to give a simple proof of the nonexistence of a metric $d$ of the type described above.

By a normal subdivision of a closed interval $[a, b]$ we mean the subdivision formed by inserting the points of the sequence $x_{1}$ $=(a+b) / 2, x_{2}=\left(x_{1}+b\right) / 2, x_{3}=\left(x_{2}+b\right) / 2, \cdots$. If $n$ is a positive integer and $\epsilon>0$, then we say that a function $f$ is of type $(n, \epsilon)$ on $[a, b]$ if the domain of $f$ contains $[a, b]$ and if the graph of $f \mid[a, b]$ consists of the broken line which joins successively the points with coordinates $(a, 0),\left(x_{n}, 0\right),\left(x_{n+1}, \epsilon\right),\left(x_{n+2}, \boldsymbol{\epsilon}\right),\left(x_{n+3}, 0\right)$, and $(b, 0)$.

We now define $S_{1}$ to be the normal subdivision of $[0,1]$. If a subdivision $S_{n}$ of $[0,1]$ has been defined, we define $S_{n+1}$ to be the refinement of $S_{n}$ which is obtained by making a normal subdivision of each interval of $S_{n}$.

We now let $T$ be the collection of all intervals $J$ such that for some $n, J$ is an interval of the subdivision $S_{n} . T$ is countable, and hence we may let $k$ be a one-to-one function whose domain is $T$ and whose range is the set of all positive integers.

Let $n$ and $m$ be positive integers. We now define the function $f_{n, m}$. If $J$ is an interval of the subdivision $S_{n}$, we define $\epsilon_{J}=1$ if $k(J)$ $\leqq m$ and $\epsilon_{J}=1 / k(J)$ if $k(J)>m$. Let $f_{n, m}$ be the function on $[0,1]$

Received by the editors November 28, 1949 and, in revised form, January 3, 1950.

${ }^{1}$ This fallacy was pointed out to the author by R. E. Priest. 
which is of type $\left(m, \epsilon_{J}\right)$ on each interval $J$ of $S_{n}$. The graph of $f_{n, m}$ has a hump on each interval of $S_{n}$. It is easily seen that $f_{n, m}$ is continuous, however, since if $\delta>0$ then the graph of $f_{n, m}$ can contain at most a finite number of humps of height greater than $\delta$.

Let $n$ be a positive integer and suppose $0 \leqq t \leqq 1$. It is easy to see that $f_{n, m}(t)=0$ for all but at most three values of $m$. Thus, we obtain $\lim _{m \rightarrow \infty} f_{n, m}(t)=0$.

Now let $f_{0}$ be the function which is identically zero on $[0,1]$. Suppose a metric $d$ exists which defines convergence to be pointwise convergence. We obtain $\lim _{m \rightarrow \infty} d\left(f_{n, m}, f_{0}\right)=0$ for each positive integer $n$. It is possible to choose integers $N_{n}$ such that if $m_{n}>N_{n}$ then $d\left(f_{n, m_{n}}, f_{0}\right)<1 / n$. It follows that any such sequence $\left\{f_{n, m_{n}}\right\}$ converges pointwise to $f_{0}$. We obtain a contradiction by showing that this is not the case.

Let $J_{1}$ be an interval of $S_{1}$ and choose $m_{1}>\max \left(k\left(J_{1}\right), N_{1}\right)$. We see that $f_{1, m_{1}}$ is 1 on some interval $J_{2}$ of $S_{2}, J_{2} \subset J_{1}$. Next choose $m_{2}>\max \left(k\left(J_{2}\right), N_{2}\right)$. It follows that $f_{2, m_{2}}$ is 1 on some interval $J_{3}$ of $S_{3}, J_{3} \subset J_{2}$. Then choose $m_{3}>\max \left(k\left(J_{3}\right), N_{3}\right)$. The procedure is clear. We obtain a nested sequence $J_{1} \supset J_{2} \supset J_{3} \supset$. . of closed intervals and integers $m_{n}>N_{n}$ such that $f_{n, m_{n}}$ is 1 on $J_{n+1}$. There is a point $p$ which belongs to each interval $J_{n}$. We now obtain

$$
\lim _{n \rightarrow \infty} f_{n, m_{n}}(p)=1 \neq 0=f_{0}(p) .
$$

This proves that $\left\{f_{n, m_{n}}\right\}$ does not converge pointwise to $f_{0}$.

UNIVERSITY OF ILLINOIS 